Musées, Patrimoine et Culture scientifiques et techniques

$134 \mid 2011$

mars - avril 2011

\title{
L'émotion a sa place dans toutes les expositions
}

Emotions have their place in all exhibitions

\section{David Sander et Carole Varone}

Édition électronique
URL : http://journals.openedition.org/ocim/840

DOI : $10.4000 /$ ocim. 840

ISSN : 2108-646X

Éditeur

OCIM

Édition imprimée

Date de publication : 1 mars 2011

Pagination : 22-28

ISSN : 0994-1908

Référence électronique

David Sander et Carole Varone, "L'émotion a sa place dans toutes les expositions », La Lettre de l'OCIM [En ligne], 134 | 2011, mis en ligne le 01 mars 2013, consulté le 19 avril 2019. URL : http:// journals.openedition.org/ocim/840; DOI : 10.4000/ocim.840

Ce document a été généré automatiquement le 19 avril 2019

Tous droits réservés 


\title{
L'émotion a sa place dans toutes les expositions
}

\author{
Emotions have their place in all exhibitions
}

David Sander et Carole Varone

Nous tenons à remercier les institutions et les personnes qui travaillent dans les musées genevois et qui ont soutenu et participé au succès de ces très enrichissantes expériences de transfert des connaissances pour le PRN Sciences affectives, en particulier : Pierre-André Loiseau, Didier Roguet et Gisèle Visinand (Conservatoire et Jardin botaniques de Genève), Véronique Bernard (guide conférencière), Danielle Decrouez (muséum d'Histoire naturelle de la Ville de Genève), Bill Ewing (ancien directeur du musée de l'Elysée Lausanne, actuellement Foundation for the Exhibition of Photography), Nathalie Herschdorfer, (anciennement curatrice au musée de l'Elysée, actuellement curatrice à la Foundation for the Exhibition of Photography), Roger Mayou et Sandra Sunier (musée international de la Croix-Rouge et du Croissant-Rouge).

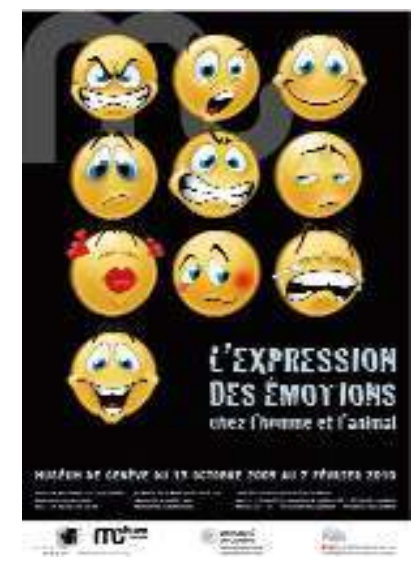

Affiche de l'exposition L'expression des émotions chez l'Homme et l'Animal, présentée au muséum d'Histoire naturelle de Genève du 12 octobre 2009 au 7 février 2010.

(c) Muséum d'Histoire naturelle de Genève

1 Même si l'émotion du visiteur n'est pas toujours prévue ni même souhaitée par les acteurs culturels en charge d'une exposition, elle est bien souvent au rendez-vous lors de 
l'interaction entre une œuvre et le public. S'interrogeant sur la place de l'émotion dans l'exposition, notre article abordera tout d'abord rapidement le thème de l'émotion en tant qu'objet d'une exposition puis s'intéressera de manière plus détaillée à la relation réciproque entre l'émotion et l'exposition: l'exposition au service de l'émotion versus l'émotion au service de l'exposition. Certaines collaborations récentes entre le Centre interfacultaire en Sciences affectives de l'université de Genève et des musées genevois illustreront notre propos.

\section{L'émotion en tant que thème d'exposition}

Chaque visiteur étant naturellement motivé par un thème d'exposition qui le concerne directement tout en étant complexe, l'émotion représente un thème d'exposition susceptible de captiver le public. Les dimensions individuelles, sociales, culturelles, artistiques, scientifiques ou encore historiques de l'émotion permettent d'ailleurs de l'aborder sous une grande variété d'angles. Par exemple, une émotion particulière traitée de manière interdisciplinaire a été l'objet de l'exposition Mélancolie : génie et folie en Occident. Cette exposition s'est tenue à Paris au Grand Palais du 13 octobre 2005 au 16 janvier 2006. La mélancolie est une source d'expression des sentiments qui a beaucoup inspiré les artistes et ce, de la période antique aux temps modernes. Récemment, un aspect général de l'émotion a été traité sous un angle historique et scientifique au muséum de Genève dans le cadre de l'exposition L'expression des émotions chez l'homme et l'animal basée sur l'œuvre de Charles Darwin. Au-delà de représenter l'émotion pour le visiteur, l'exposition est également, comme nous allons le voir dans la prochaine section, un lieu qui induit des émotions.

\section{Emotions dans les musées genevois}

Le Pôle de Recherche National en Sciences affectives, financé sur 12 ans par la Confédération Helvétique, se caractérise par une recherche de pointe ainsi que par la valorisation des résultats scientifiques auprès d'un large public. Le PRN Sciences affectives a ainsi pris l'initiative d'inviter divers musées genevois afin de réaliser, en interaction avec eux, plusieurs projets de transfert de connaissance.

Émotions de collections, collections d'émotions présentée au conservatoire et jardin botaniques de Genève du 1er mai au 4 octobre 2009. Cette exposition explorait les émotions suscitées par une collection de plantes auprès du botaniste averti et du public béotien. Le parcours scénographique faisait appel aux cinq sens. Il répartissait les collections en fonction des émotions que certaines plantes auraient tendance à inspirer, dont par exemple la peur associée aux plantes carnivores.

L'expression des émotions chez l'homme et l'animal proposée par le muséum d'Histoire naturelle de Genève, du 12 octobre 2009 au 7 février 2010. Basée sur l'ouvrage de Darwin publié en 1872, cette exposition a notamment traité de la vie émotionnelle des animaux, des ressemblances existantes avec les comportements humains et animaux ou encore de l'influence de la culture sur le registre des expressions émotionnelles. Le PRN en Sciences affectives a animé sept ateliers, pour les enfants de 5 à 12 ans, sur l'importance de nos expressions verbales dans les 
relations sociales. Cette exposition, accompagnée d'une brochure en anglais et en français, a ensuite été présentée au Fort de l'Écluse du 15 juin au 15 septembre 2010.

Stigmates du 4 mars au 26 juillet 2009 réalisée par le musée international de la Croix-Rouge et du Croissant-Rouge et le musée de l'Elysée à Lausanne. Les travaux de sept photographes contemporains ont convié le visiteur à porter un regard réflexif sur diverses situations liées à la souffrance, comme par exemple les visages des soldats américains en permission avant de retourner en Irak, les portraits des albinos africains ou les images de maisons dévastées par l'ouragan Katrina. Un neuropsychologue et des historiens des religions ont posé des regards différents sur les photographies de l'exposition. Un ouvrage sur cette thématique est en cours d'édition chez Thames\&Hudson.

\section{L'exposition au service de l'émotion}

3 Les expositions peuvent avoir pour fonction de déclencher des émotions. Par exemple, dans les musées d'art, les émotions, que ce soient celles des artistes, celles qui sont présentes dans les œuvres, ou celles des visiteurs, sont typiquement les bienvenues. Une certaine catégorie d'émotion qui est spécifique à l'art est même largement étudiée en sciences affectives : les émotions esthétiques (Robinson, 2005). Cette gamme d'émotions a acquis ses lettres de noblesse au sein des institutions muséales, et une exposition d'art visuel, littéraire ou musical qui déclencherait le «Syndrome de Stendhal» chez ses visiteurs serait certainement considérée comme une réussite. Le "Syndrome de Stendhal » fait référence à la réaction émotionnelle que Stendhal avait eu devant la beauté sublime de l'art telle qu'il l'a décrit dans Rome, Naples et Florence : «J'étais arrivé à ce point d'émotion où se rencontrent les sensations célestes données par les Beaux Arts et les sentiments passionnés. En sortant de Santa Croce, j'avais un battement de cœur, la vie était épuisée chez moi, je marchais avec la crainte de tomber »

(es visiteurs s'oriente globalement dans deux directions (Silvia, 2005). La première s'intéresse à établir ce qui, dans l'œuvre d'art, est susceptible de déclencher une émotion. Ainsi, par exemple, dans l'art visuel, l'on étudie les couleurs, les symétries, ou encore les proportions. La seconde direction s'intéresse à établir ce qui, chez le visiteur, peut favoriser l'émergence de telle ou telle émotion esthétique. Ainsi, l'on peut s'intéresser aux normes culturelles que le visiteur a intégrées, à ses valeurs, à sa personnalité, ou encore à son expertise dans le domaine artistique en question.

Si il est admis que le déclenchement d'émotions esthétiques est un objectif déclaré des expositions artistiques, l'utilité, voire même l'acceptabilité, d'autres gammes d'émotions dans les lieux d'exposition semble en revanche plus controversée. Par exemple, une exposition historique, scientifique ou médicale qui déclencherait du dégoût, de la peur, de la colère, de la tristesse, de la honte, ou encore de la culpabilité remplirait-elle sa mission fondamentale qui est d'être une source de connaissance? Un tel débat a été particulièrement vif dans le cadre de l'exposition Our Body : à corps ouvert sur le corps humain qui a connu un véritable succès international tout en heurtant certaines sensibilités et déclenchant de vives émotions soit en faveur de l'exposition, soit contre son établissement dans telle ou telle ville. Gunther von Hagens, l'inventeur de la 
plastination, est à l'origine de cette exposition polémique qui présente des cadavres humains disséqués, dépecés et coupés en rondelles. Dans ce cas, ce n'était bien entendu pas le thème fondamental de l'exposition qui faisait débat (la connaissance de l'anatomie du corps humain), mais bien le traitement de ce thème et les doutes sur la manière dont les corps humains ont été obtenus qui déclenchaient des émotions telles que la colère ou le dégoût à la fois sur le principe lui-même d'une telle exposition mais également lors de l'expérience de visite.

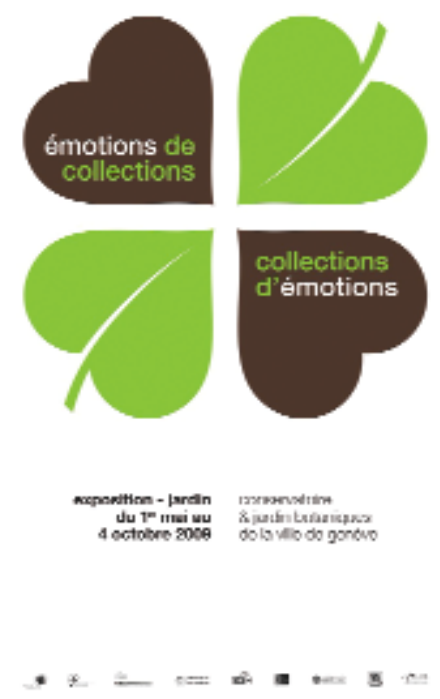

Affiche de l'exposition Émotions de collections, collections d'émotions, présentée au Conservatoire et Jardin botaniques de Genève du Ter mai au 4 octobre 2009

(C) Conservatoire et Jardin botaniques de Genève

Dans le cas de Our Body : à corps ouvert, il est probable que la dimension émotionnelle de l'exposition n'a pas été ignorée par ses concepteurs. Mais, même si cela n'est pas anticipé, de manière générale, une exposition peut déclencher une vaste palette d'émotions. Ainsi des émotions dites parfois "utilitaires » (par exemple la peur, le dégoût, la colère ou la tristesse, la joie) ou des émotions morales (par exemple la honte, la culpabilité, la fierté) sont, à des intensités variables, des éléments incontournables de l'expérience de visite. Ainsi, une exposition sur le Sida ne va-t-elle pas déclencher de la peur compte tenu du danger que représente cette maladie? Une exposition sur le racisme ne va-t-elle pas déclencher de la colère si l'on considère l'injustice qu'une telle attitude représente? Une exposition sur la collaboration lors de la déportation des juifs en France ne va-t-elle pas déclencher de la honte chez un visiteur français étant donnée l'atteinte à ses valeurs morales par des membres de sa propre nation? En revanche, une exposition sur la Résistance durant la même période historique ne va-t-elle pas déclencher de la fierté chez ce même visiteur étant donné le renforcement de ses propres valeurs morales par des membres de sa propre nation? Une exposition sur les victimes de catastrophes humanitaires ne va-t-elle pas déclencher de la sympathie étant donnée la souffrance endurée par ces personnes qui n'en sont pas responsables (voir l'encadré concernant l'exposition Stigmates au musée international de la Croix-Rouge et du Croissant-Rouge). 


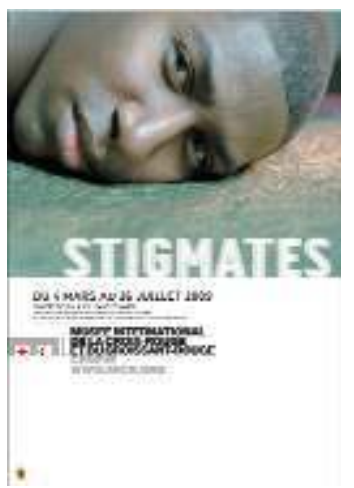

Affiche de l'exposition Stigmates, présentée au musée international de la Croix-Rouge et du CroissantRouge du 4 mars au 26 juillet 2009

(c) Musée international de la Croix-Rouge et du Croissant-Rouge

De telles expositions, sans être à la recherche de sensationnel ni orientées vers une culture du spectacle, vont donc déclencher des émotions. Mais, faut-t-il s'en inquiéter en opposant la connaissance à l'émotion? Au contraire, les recherches en sciences affectives indiquent que cette opposition, qui trouve son origine épistémologique dans une opposition " raison versus passion » puis dans une opposition « cognitif versus affectif », n'est pas empiriquement justifiée (Sander \& Scherer, 2009). En particulier, les théories actuelles de l'émotion insistent sur la dimension cognitive de l'émotion. Ces théories, que l'on appelle théories de l'évaluation cognitive, proposent d'expliquer un aspect fondamental de l'émotion qui est négligé par les autres théories: le déclenchement de l'émotion. Ainsi, ces théories se focalisent sur les mécanismes cognitifs d'interprétation de l'événement par lequel l'émotion va naître, se déployer dans le temps, et se différencier, c'est-à-dire devenir une émotion particulière comme la peur, la honte ou la joie. L'évaluation cognitive (appraisal) de tout événement s'effectue sur la base de critères qui déterminent à la fois la nature et l'intensité de l'émotion. De façon générale, cette approche explique pourquoi seuls les événements pertinents pour les buts, les besoins, ou les valeurs d'un individu donné à un moment donné sont susceptibles de déclencher une émotion. C'est certainement ainsi que toute exposition a le potentiel de déclencher telle émotion chez un visiteur donné à un moment donné mais telle autre émotion (ou aucune émotion!) chez un autre visiteur au même moment ou encore chez le même visiteur à un autre moment. Cette subjectivité dans le déclenchement d'une émotion met justement en avant l'importance de la variabilité intra et inter individuelle durant l'expérience de visite. Cependant, même si de nombreuses variables (comme l'âge, le genre, la personnalité, l'état de stress, les buts du moment, les valeurs culturelles, la visite seul ou en groupe) peuvent modifier la réaction émotionnelle face à une même exposition, cela ne veut en aucun cas dire que l'émotion est irrationnelle et imprévisible. La logique d'interprétation qui régit l'évaluation cognitive et les fonctions adaptées de la réponse émotionnelle font qu'il est en général possible, si l'on dispose de suffisamment d'éléments, de prédire les réactions qui peuvent être typiquement déclenchées par une situation donnée au sein des composantes de l'émotion que sont l'évaluation cognitive, l'activité physiologique périphérique, l'activité motrice, la tendance à l'action, et le sentiment subjectif.

\section{Définition d'une émotion}


Qu'est-ce qu'une émotion? Les émotions sont souvent décrites dans un cadre incluant d'autres phénomènes affectifs tels les sentiments, la motivation, l'humeur, la passion, le style affectif, la réactivité affective ou encore les pulsions. Certains de ces phénomènes dépendent les uns des autres, et le fait d'individualiser certains phénomènes affectifs, notamment les émotions, représente un défi scientifique. Les différentes classes de définitions ont souvent mis l'accent sur des aspects bien particuliers de l'émotion comme, par exemple, la dimension subjective du sentiment, les catégories de stimuli déclencheurs, les mécanismes physiologiques impliqués, l'expression des comportements, les effets adaptatifs, ou encore les effets perturbateurs de l'émotion. De nos jours, en réponse à la question « qu'est-ce qu'une émotion? ", la plupart des théoriciens de l'émotion s'accordent à considérer les émotions comme « des épisodes de changements corporels et psychiques qui sont coordonnés dans plusieurs composantes de l'organisme (incluant l'activation physiologique, l'expression motrice et le sentiment subjectif et, également, les tendances à l'action et les processus cognitifs), en réponse à des événements internes (par exemple des pensées ou des souvenirs) ou externes (par exemple le comportement d'autrui, un changement dans la situation, la rencontre d'un nouveau stimulus) qui sont d'une signification majeure pour l'organisme ». Cette définition capture les points jugés indissociables de l'émotion, notamment sa nature épisodique. L'émotion est un épisode marqué par un changement du fonctionnement de l'organisme en réponse à un événement externe ou interne. Ce changement dure un certain moment (typiquement quelques secondes ou minutes), diminue d'intensité au cours du temps et s'éteint plus ou moins rapidement. Un autre point majeur abordé par cette définition est l'idée que les émotions sont normalement générées par des événements qui ont une signification importante pour l'organisme. De ce fait, elles peuvent être conçues comme des « détecteurs de pertinence » qui évaluent les stimuli et l'environnement par rapport à leur signification pour le sujet. La plupart des théoriciens acceptent l'idée que la nature de cette évaluation va déterminer à la fois la réponse fonctionnelle de l'organisme et la nature des changements organiques et mentaux qui se produiront durant l'épisode émotionnel.

8 Ainsi, une exposition qui révèle un discours, adopte une approche, élabore une scénographie, s'engage dans une démarche de médiation et produit des connaissances est également une source d'émotions de natures et d'intensités variables. Certes, des émotions esthétiques peuvent être induites, mais des émotions « utilitaires » ou morales peuvent également l'être. Une autre gamme d'émotions qui, peut-être plus que toute autre, a pour objet l'exposition est celle que l'on appelle parfois «émotions épistémiques » dont une émotion bien connue des professionnels d'expositions est la plus représentative : l'intérêt (Silvia, 2006). La fonction de ces émotions épistémiques est de favoriser la connaissance. Par exemple, la fonction principale de l'intérêt est de motiver l'exploration de la nouveauté et de faciliter l'apprentissage. Ainsi, il semble évident que la plupart des expositions cherchent à déclencher et entretenir l'intérêt des visiteurs. Comme nous venons de le voir, dans le cas de l'intérêt, comme dans celui des autres émotions, l'on peut dire que l'exposition est au service de l'émotion car l'exposition est source d'émotions, elle les déclenche. Mais, comme nous le verrons dans la section suivante, c'est également l'émotion qui est au service de l'exposition en facilitant la réalisation des fonctions muséales. 


\section{L'émotion au service de l'exposition}

9 S'intéresser à la manière dont l'émotion peut être au service d'une exposition implique de s'intéresser aux effets de l'émotion sur l'esprit et, plus spécifiquement, sur la manière dont les fonctions psychologiques recrutées lors d'une exposition peuvent être facilitées ou, au contraire, entravées par l'émotion. Malgré la diversité des émotions (par exemple la joie, la peur, la colère, la tristesse, le dégoût, la surprise, la honte, la fierté ou l'émerveillement), des principes généraux ont été proposés pour comprendre ce que sont les émotions (l'encadré ci-contre). L'émotion est considérée par de nombreuses théories actuelles comme ayant un statut privilégié au sein de l'esprit humain. En effet, les études empiriques récentes démontrent que l'émotion peut faciliter de nombreux mécanismes cognitifs tels que la perception, l'attention, la mémoire, la prise de décision, ou encore le jugement moral. Dans la mesure où ces mécanismes sont parmi ceux qui sont essentiellement mobilisés par le visiteur d'une exposition, il semble important de mieux prendre en compte le rôle des émotions dans une exposition.

10 L'objectif de notre article ne nous conduit pas à explorer ici l'ensemble des liens entre l'émotion et d'autres fonctions cognitives importantes pour une exposition, mais nous pouvons considérer plus particulièrement les mécanismes attentionnels et les mécanismes mnésiques car ils sont certainement parmi les plus importants pour une exposition. De nombreuses variables modulent notre attention. Par exemple, le fait de volontairement se concentrer va, bien entendu, modifier l'attention que l'on porte à tel ou tel élément d'une exposition. Un autre type d'attention qui est appelé «l'attention émotionnelle » (Vuilleumier, 2005) a été décrit pour rendre compte du fait que les stimuli émotionnels, qu'ils soient agréables ou désagréables, capturent l'attention et la retiennent. Cette « attention émotionnelle » automatique fonctionne très rapidement et oriente ainsi en partie l'activité du visiteur indépendamment de sa conscience. Ainsi, un objet qui est émotionnel augmente l'attention du visiteur envers cet objet précis, facilitant l'accès aux informations relatives à cet objet. Suivant la même logique, certains auteurs ont décrit une "mémoire émotionnelle " qui représenterait les événements émotionnels de manière privilégiée par rapport aux événements dits neutres (Labar et Cabeza, 2006).

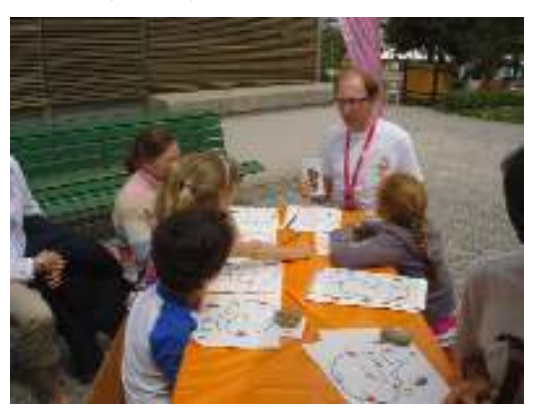

Atelier avec des enfants concernant le cerveau émotionnel lors de l'exposition Émotions de collections, collections d'émotions.

(c) Carole Varone

11 De manière classique, l'on peut distinguer trois grandes étapes mnésiques lorsque le visiteur parcourt une exposition. Prenons l'exemple d'un visiteur qui perçoit un tableau dans une salle d'exposition. Cette perception visuelle, qui est intégrée à un contexte particulier de visite, peut être traitée de manière plus ou moins approfondie et intense 
par le cerveau et « entrer » en mémoire. Bien entendu, le niveau d'attention que l'on aura porté au tableau en question modifie cette "entrée » en mémoire; en particulier, si le tableau est pertinent pour l'individu - parce qu'il exprime par exemple un thème qui le touche fortement -, alors l'attention émotionnelle se déploierait et faciliterait ainsi l'encodage. C'est notamment pour cela que l'on pense que dès «l'entrée » en mémoire la nature émotionnelle d'une information rendrait celle-ci privilégiée. Suite à cette étape, des informations concernant le tableau et l'expérience particulière qu'il a provoquée sont stockées en mémoires. Durant cette étape, les événements sont consolidés, ce qui permet de les retenir même parfois à très long terme. Certaines études suggèrent que cette étape de consolidation profite encore plus aux événements émotionnels qu'aux événements neutres, permettant ainsi une sélection des informations retenues. Finalement, telle qu'évoquée dans le célèbre épisode de «la madeleine de Proust », l'étape qui est la plus souvent associée à l'émotion est celle dite de "rappel». Cette étape de «sortie » d'un souvenir peut être très fortement influencée par la valeur émotionnelle de l'événement rappelé. D'ailleurs, la précision subjective, l'intensité et la nature parfois très « vive » de certains de ces rappels peut même déclencher une autre émotion (la nostalgie, par exemple). Certains visiteurs pourraient donc, sur la base des effets de l'émotion sur la mémoire, décrire une visite, même scientifique, comme étant inoubliable.

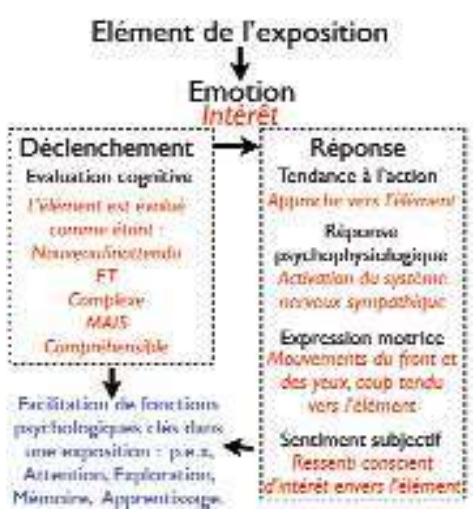

Le déclenchement émotionnel et la réponse émotionnelle avec comme exemple l'émotion épistémique « Intérêt » qui est typiquement déclenchée lorsqu'un élément d'une exposition est évalué comme étant nouveau/original et complexe tout en étant compréhensible. La réponse émotionnelle correspond alors à une approche vers l'élément (tendance à l'action), une activation du système nerveux sympathique (réponse psychophysiologique), des mouvements du front et des yeux avec le coup tendu vers l'élément (expression motrice) et un ressenti conscient d'intérêt envers l'élément en question (sentiment subjectif). Basé sur Silvia (2006).

Comme évoqué plus haut, une émotion clé qui est admise par tous comme étant au service de l'exposition est l'intérêt. Pour déclencher l'intérêt vis-à-vis d'un élément muséal, il faut augmenter trois variables à la fois : la nouveauté/originalité de l'élément, sa complexité, et sa compréhension (Silvia, 2006). Si la nouveauté et la complexité sont augmentées mais que la visite ne permet pas une meilleure compréhension, alors l'émotion épistémique induite serait plutôt de la confusion que de l'intérêt (Silvia, 2006) qui peut également avoir des effets très positifs sur l'exploration et la recherche de connaissances. La prise en compte de ces paramètres de nouveauté/originalité, complexité, et compréhension reflète bien l'importance de l'expertise dans l'interprétation des expositions. Par exemple, un expert en art contemporain sera certainement plus à même de déceler l'originalité d'une nouvelle œuvre, d'en apprécier la complexité tout en la comprenant, et la trouverait donc plus intéressante qu'un novice. Ainsi, l'expertise du collectionneur lui permet de reconnaître une pièce comme étant 
intéressante. Il semble que le même processus soit en place lorsque le visiteur éprouve de l'intérêt. La réponse émotionnelle durant l'intérêt correspond à un ensemble de modifications au sein des composantes émotionnelles (tendance à l'action, réponse psychophysiologique, expression motrice, et sentiment subjectif). L'intérêt n'est pas une émotion qui se limite à certains domaines d'expositions plaisants car, même si l'intérêt est une émotion agréable à ressentir, il semble qu'elle ne soit pas liée à la nature agréable du stimulus lui-même. En effet, même des objets potentiellement dégoûtants, comme par exemple des corps humains décharnés, peuvent susciter de l'intérêt.

En conclusion, il semble que le lieu d'exposition soit au service des fonctions émotionnelles et que, réciproquement, l'émotion peut être considérée comme une alliée pour remplir les fonctions muséales. Cependant, pour que l'émotion soit vraiment au service de l'exposition, un point important à considérer est celui de la convergence des deux objets, celui de l'exposition et celui de l'émotion. En effet, une incohérence entre les émotions déclenchées et les intentions des différents corps de métiers impliqués dans une exposition pourrait avoir des effets négatifs. Un tel problème serait rencontré dans des cas où un double objet serait présent au sein de l'exposition, générant ainsi une interférence potentielle. Par exemple si le propos lui-même, qui est l'objet de l'exposition, est incompatible avec la scénographie, qui pourrait être l'objet principal de l'émotion, alors, dans ce cas, l'émotion ne serait pas au service du propos mais, au contraire, en limiterait l'accès. Ainsi, si les effets positifs de l'émotion qui font notamment que l'attention et la mémoire des visiteurs sont préférentiellement allouées à des événements émotionnels se produisent pour des éléments périphériques au propos, il y a un risque pour que cela se fasse au détriment de l'attention et de la mémoire allouées au propos luimême. En bref, la nature émotionnelle d'un élément lui donne un statut privilégié, et un privilège se fait toujours au dépend d'autres éléments. Donc, pour que les effets positifs de l'émotion profitent aux propos de l'exposition, il est préférable de rendre émotionnel ce qui est directement pertinent aux propos; sinon le processus risque d'être contreproductif.

Nous avons seulement considéré ici l'émotion du visiteur, mais qu'en est-il de l'émotion créatrice des acteurs culturels? Les émotions à considérer lors de la planification d'un thème exposition sont-elles les mêmes que celles qui sont à considérer lors de la conception de l'exposition, de la mise en place de la scénographie ou encore du développement des activités de médiation? Une coordination des différents corps de métiers sur ces questions pourrait augmenter l'efficacité des expositions. Il nous semble que si la prise en compte de l'émotion est considérée à chaque étape de l'exposition, de la conception à la médiation, les fonctions de l'émotion et les fonctions muséales peuvent alors se renforcer mutuellement en démontrant que l'émotion a sa place dans toutes les expositions. 


\section{BIBLIOGRAPHIE}

Labar, K.-S. et Cabeza, R. Cognitive neuroscience of emotional memory, Nature Reviews Neuroscience, $\mathrm{n}^{\circ} 7,2006$, pp. 54-64.

Oatley, K. Creative expression and communication of emotions in the visual and narrative arts, in Davidson, R.-J. Scherer, K.-R. and Goldsmith, H.-H. (eds), Handbook of affective sciences. New York and Oxford: Oxford University Press, 2003, pp. 481-502.

Robinson, J. Deeper than reason: emotion and its role in literature, music, and art. New York and Oxford: Oxford University Press, 2005.

Sander, D. et Scherer, K.-R. Traité de psychologie des émotions. Paris : Dunod, 2009.

Sander, D. et Scherer, K.-R. (dir.) The Oxford Companion to Emotion and the Affective Sciences. New York and Oxford: Oxford University Press, 2009.

Silvia, P.-J. Emotional responses to art: from collation and arousal to cognition and emotion, Review of General Psychology, ${ }^{\circ}$ 9, 2005, pp. 342-357.

Silvia, P.-J. Exploring the psychology of interest. New York and Oxford: Oxford University Press, 2006 Vuilleumier, P. How brains beware: neural mechanisms of emotional attention, Trends in Cognitive Sciences, $\mathrm{n}^{\circ}$ 9, 2005, pp. 585-594.

\section{RÉSUMÉS}

En présentant les liens théoriques et pratiques entre l'étude scientifique de l'émotion et l'expérience de visite au musée, cette contribution montre - à travers l'examen des différents paramètres de déclenchement de l'émotion chez le visiteur - comment le lieu d'exposition est au service des fonctions émotionnelles et à l'inverse comment l'émotion aide le lieu à remplir ses fonctions muséales.

By presenting theoretical and practical links between scientific studies of emotion and the museum visit experience, this combination shows - via the study of different 'emotional triggerparameters in visitors - how the exhibition site is on the one hand at the service of emotional functions, and how on the other hand emotions help the visitor space to fulfil its function.

\section{INDEX}

Mots-clés : émotion, visiteurs, étude de publics

\section{AUTEURS}

\section{DAVID SANDER}

David Sander est professeur à la section de Psychologie de l'université de Genève, directeur du Laboratory for the study of Emotion Elicitation and Expression et 
coordinateur scientifique du Pôle de Recherche National suisse en Sciences affectives à

l'université de Genève. david.sander@unige.ch

\section{CAROLE VARONE}

Carole Varone est responsable du transfert des connaissances au Pôle de Recherche National suisse en Sciences affectives à l'université de Genève 